\title{
HIGH-FIDELITY FINITE ELEMENT MODELING AND ANALYSIS OF ADAPTIVE GAS TURBINE STATOR-ROTOR FLOW INTERACTION AT OFF-DESIGN CONDITIONS
}

\author{
Nikita Kozak $^{1} \quad$ Fei Xu $^{1,2} \quad$ Manoj R. Rajanna $^{1} \quad$ Luis Bravo $^{3} \quad$ Muthuvel Murugan $^{3}$ \\ Anindya Ghoshal ${ }^{3} \quad$ Yuri Bazilevs $^{4} \quad$ Ming-Chen Hsu $^{{ }^{*}}$ \\ ${ }^{1}$ Department of Mechanical Engineering, Iowa State University \\ Ames, Iowa 50011, USA \\ ${ }^{2}$ Ansys Inc., \\ Austin, Texas 78746, USA \\ ${ }^{3}$ U.S. Army Research Laboratory \\ Aberdeen Proving Ground, \\ Maryland 21005, USA \\ ${ }^{4}$ School of Engineering, Brown University \\ Providence, Rhode Island 02912, USA
}

\begin{abstract}
The objective of this work is to computationally investigate the impact of an incidence-tolerant rotor blade concept on gas turbine engine performance under off-design conditions. When a gas turbine operates at an off-design condition such as hover flight or takeoff, large-scale flow separation can occur around turbine blades, which causes performance degradation, excessive noise, and critical loss of operability. To alleviate this shortcoming, a novel concept which articulates the rotating turbine blades simultaneous with the stator vanes is explored. We use a finite-element-based moving-domain computational fluid dynamics (CFD) framework to model a single high-pressure turbine stage. The rotor speeds investigated range from $100 \%$ down to $50 \%$ of the designed condition of $44,700 \mathrm{rpm}$. This study explores the limits of rotor blade articulation angles and determines the maximal performance benefits in terms of turbine output power and adiabatic efficiency. The results show articulating rotor blades can achieve an efficiency gain of $10 \%$ at off-design conditions thereby providing critical leap-ahead design capabilities for the U.S. Army Future Vertical Lift (FVL) program.
\end{abstract}

Keywords: compressible flow, stabilized finite element methods, gas turbine, off-design performance

\section{INTRODUCTION}

Although the development of a gas turbine engine is considered mature, the ever-higher power and efficiency requirements needed to advance today's propulsion systems call for innovation [1-3]. One promising development to address this call is the variable speed power turbine (VSPT), which would be able to meet the requested power and efficiency levels in a wider range of operating conditions. A VSPT would be able to alter its operating speed without facing the substantial performance losses that today's engines experience at off-design conditions [1, 4]. An off-design condition is an operating speed that the engine was not designed to operate at, typically any speed other than $100 \%$. The cause of performance losses at off-design operation stems from the aerodynamic flow separation around the turbine blades. The VSPT can be specifically applicable to the U.S. Army Future Vertical Lift (FVL) effort, where it would be deployed to overcome the challenges associated with off-design operations.

The idea of VSPT could be enabled by a novel incidencetolerant rotor blade concept [5] known as the adaptive turbomachinery blade [6]. Adaptive turbomachinery blades would maintain optimal flow patterns and therefore, achieve satisfactory performance in a wide range of operating conditions $[2,3]$. These flow patterns will be maintained by synchronously articulating the turbine's stator and rotor blades to limit the presence of flow separation. Figure 1(a) illustrates the exterior actuators which would enable stator articulation. This use of exterior

${ }^{*}$ Corresponding author (jmchsu@iastate.edu) 


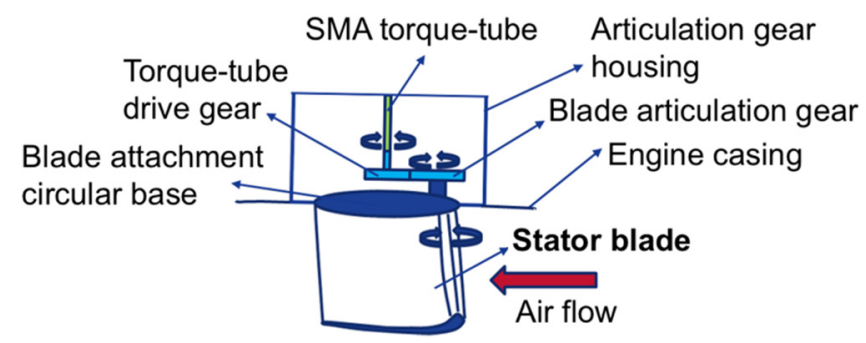

(a) Stator articulation

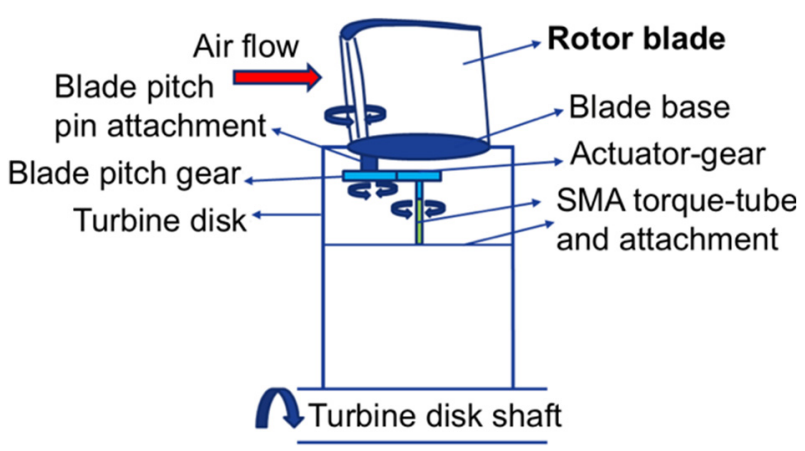

(b) Rotor articulation

Fig. 1 Proposed stator and rotor articulation mechanisms in Murugan et al. [6].

actuators is common for articulating the compressor inlet vanes [7-9]. However, rotor articulation utilizes a novel concept of interior actuators. This mechanism, depicted in Figure 1(b), will be located within the turbine's shaft and features multiple actuators to pitch the blades while maintaining a balanced disk.

The lack of understanding on the complex internal flows of a gas turbine stage, specifically on unsteady flow separation, acts as a barrier in continuing the development of adaptive turbomachinery blades. This barrier stems from the reason that generic flow separation leads to the formation of dynamic-stall-like vortices whose evolution and interaction with surfaces generate inconstant forces along the airfoil blades and have a significant impact on performance $[2,3,10-12]$. These performance impacts include lowered power and efficiency as well as increased structure stress, noise and likelihood of stall and shocks $[2,3,10]$.

Previous experimental and numerical investigations $[13,14]$ have focused on understanding the aerodynamic phenomena related to blade articulation within the compressor section of the gas turbine engine. Due to the lack of mechanism for rotor blade articulation, these studies provided limited information on rotor blade articulation. Therefore, to overcome this knowledge barrier and further the development of adaptive blade technology, this work utilizes a novel finite element based moving-domain computational fluid dynamics (CFD) framework [15] to investigate the relationship of rotor blade articulation and turbine stage flow separation and performance.

This paper is organized as follows. In Section 2, we summarize the finite element methods used in this work for the CFD analysis of a gas turbine. The turbine stage geometry, modeling setup, and variables of interest are also described. In Section 3, we present and discuss the results of a mesh convergence study and an extensive investigation on the flow fields and gas turbine performance impacted by the rotor articulation under both design and off-design conditions. Finally, the conclusions of this study and the path forward are summarized in Section 4.

\section{COMPUTATIONAL MODELING AND ANALYSIS}

\subsection{Finite element modeling}

The fluid flow inside gas turbines spans a wide range of flow regimes from subsonic to supersonic. To accommodate such a wide range of Mach numbers, a suitable CFD simulation method needs to be robust across the entire regime. In this paper, we utilize the stabilized finite element formulation for the Navier-Stokes equations of compressible flows on moving domains in an arbitrary Lagrangian-Eulerian (ALE) frame, originally developed in $\mathrm{Xu}$ et al. [15]. The formulation is stabilized using the streamline upwind/Petrov-Galerkin (SUPG) stabilization methodology [16-22] and is augmented with a discontinuity-capturing (DC) operator [23-30]. Through this combination, superior robustness and numerical stability are achieved even for extreme flow conditions at high Mach and Reynolds numbers. Velocity and temperature Dirichlet boundary conditions are enforced weakly based on Nitsche's method [31], which allows us to relax the strict boundary-layer resolution requirements for wall-bounded turbulent flows without sacrificing the overall solution accuracy [32-35]. As a result, satisfactory solutions can be obtained using relatively coarser meshes and lower computational costs in the context of full-annulus gas-turbine simulations.

Additional techniques are required to couple the relative rotational motion that is present between the stator and rotor. Following the method in $\mathrm{Xu}$ et al. [15], we partition the computational domain into the rotor and stator subdomains and couple them through the slidinginterface formulation developed therein. By applying this approach, optimal mesh quality can be maintained throughout the simulation, and we avoid the need for mesh deformation or remeshing that arises if the rotor and stator are meshed in the same domain. The sliding-interface technique was first proposed for incompressible flows in Bazilevs and Hughes [36] and has been successfully applied to wind turbine applications in Hsu et al. [37, 38]. In the case of compressible flows, the sliding-interface formulation was developed and has been applied to the simulation of a gas turbine in $\mathrm{Xu}$ et al. [15] and to the aerodynamic analysis of rotorcraft in Xu et al. [39].

Finally, all the identified numerical components are integrated into a single formulation, which is then discretized in time by an implicit generalized- $\alpha$ time-integration 


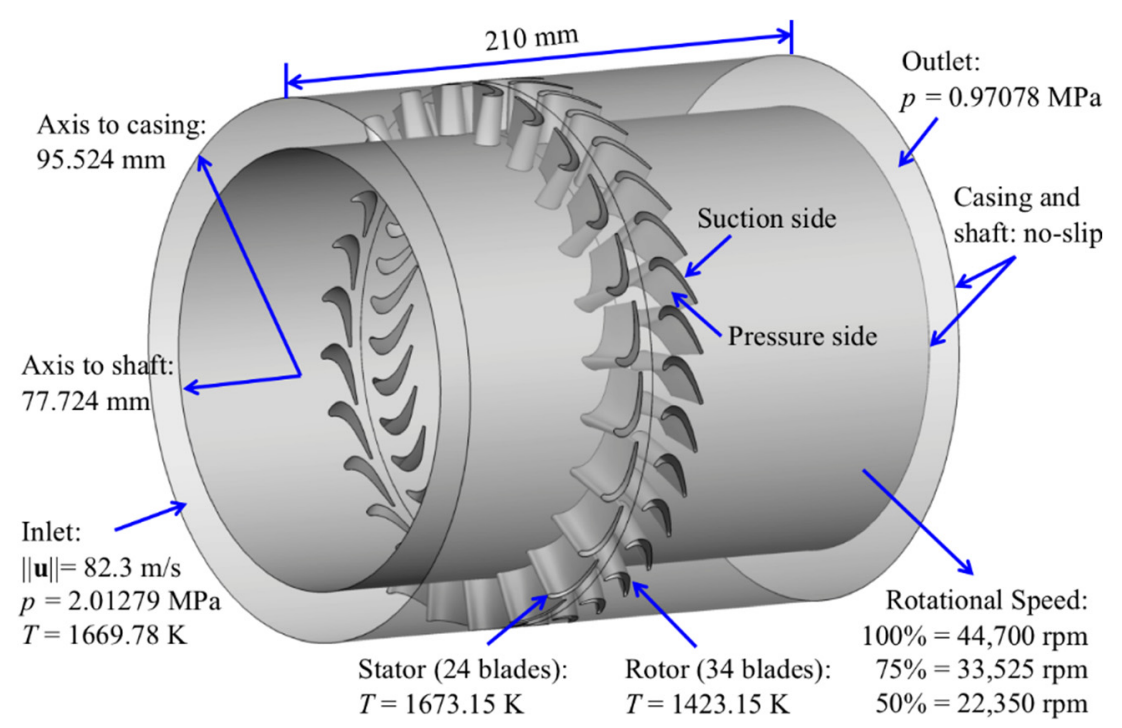

Fig. 2 Problem setup, geometry and dimensions of the turbine stage.

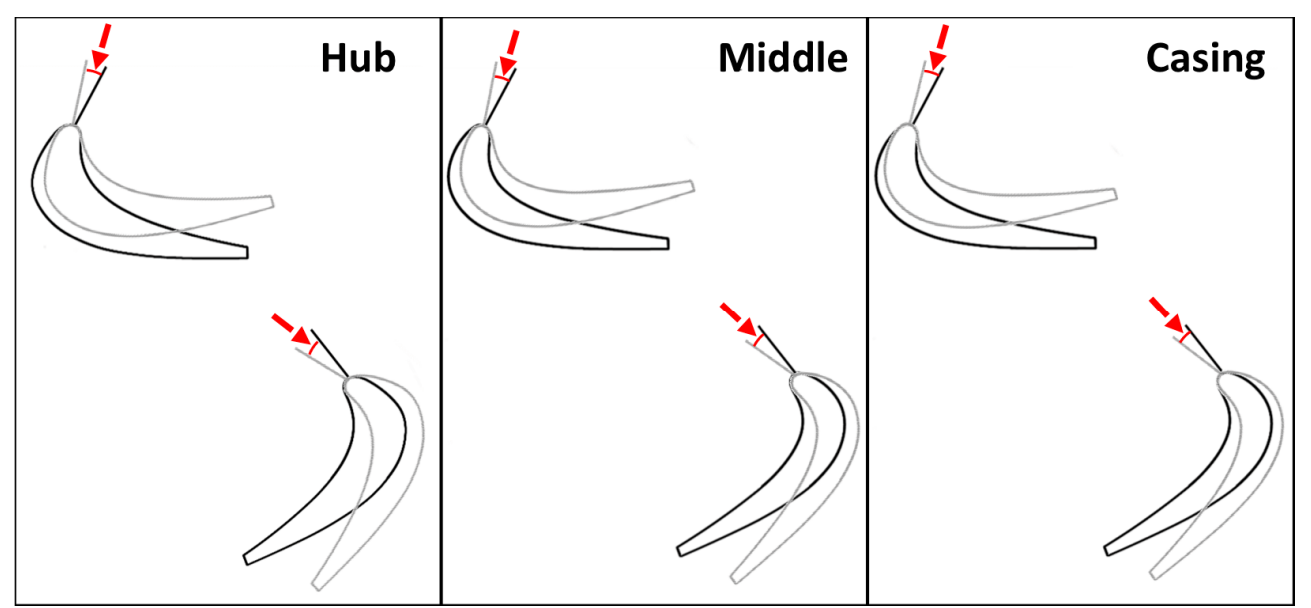

Fig. 3 Illustration of stator and rotor airfoil profiles at different blade heights and their baseline $\left(0^{\circ}\right)$ and articulated positions. Baseline positions are colored black and articulated positions are colored gray. A positive angle corresponds to a counterclockwise articulation.

scheme [40-42]. The solution of the nonlinear algebraic equation system resulting from the generalized- $\alpha$ scheme is obtained at each time step with the Newton-Raphson method. A block-diagonal preconditioned GMRES technique [43] is used to solve the linear equation system for each Newton-Raphson iteration. The numerical methods presented in this section have been extensively verified and validated in $\mathrm{Xu}$ et al. [15, 39], and we refer the readers to the cited literature for further details.

\subsection{Turbine stage simulation setup}

In this work, we investigate the articulation of rotor blades within a single high-pressure turbine stage that features similar dimensions and operating conditions to engines found in rotorcraft like Apache and Blackhawk. This turbine stage, shown in Figure 2, has a total axial length of $210 \mathrm{~mm}$, an inner shaft radius of $77.724 \mathrm{~mm}$, and a casing radius of $95.524 \mathrm{~mm}$. The annulus is composed of 24 stator and 34 rotor blades. The airfoil profiles at different blade heights are shown in Figure 3, where baseline positions $\left(0^{\circ}\right)$ are colored black and articulated positions are colored gray. The articulation of the stator and rotor blades are completed about the leading edge, where a positive angle corresponds to a counterclockwise rotation. The 3D geometric model of the turbine stage is generated using a parametric design tool proposed in $\mathrm{Xu}$ et al. [15], based on the idea of an interactive geometry modeling platform [44]. The design tool allows us to parametrically pitch the blade in the computer-aided design (CAD) model through input parameters that control the pitch angles of the rotor and stator blades. This approach enables us to efficiently carry out a series of gas-turbine simulations at different blade angles.

We consider a turbine stage designed to operate at application specific conditions to meet FVL requirements. We operate this stage at two off-design operating speeds of $22,350 \mathrm{rpm}(50 \%)$ and $33,525 \mathrm{rpm}(75 \%)$ as well as a 
design speed of 44,700 rpm (100\%). These operating speeds effectively replicate different engine conditions that are seen during cruise, hover, and takeoff, respectively [1-3]. Furthermore, we assume all the conditions operate under the same gas-turbine combustor exit flow conditions. This includes a uniform axial inflow with velocity of $82.3 \mathrm{~m} / \mathrm{s}$, temperature of 1669.78 $\mathrm{K}$, and pressure of $2.01279 \mathrm{MPa}$ at the inlet boundary. On the opposite end, the outlet boundary has a fixed pressure of $0.97078 \mathrm{MPa}$. The temperatures on the stator and rotor blades are specified as $1673.15 \mathrm{~K}$ and 1423.15 $\mathrm{K}$, respectively. Also, the inner shaft and outer casing surfaces have no-slip velocity and adiabatic conditions. The dynamic viscosity of gas is $\mu=5.551 \times 10^{-5} \mathrm{~kg} /(\mathrm{m} \cdot \mathrm{s})$. The setup of the computation is summarized in Figure 2.

\subsection{Performance analysis}

To analyze the performance of the different rotor blade angles, output power and efficiency are key quantities that provide insight on a turbine's capabilities $[45,46]$. The output power is calculated by the product of the total shaft torque and the angular rotational speed, where the total torque is obtained by integrating the local torque contribution from the fluid traction over the entire rotor surface. The adiabatic efficiency of a turbine stage is calculated using the following equation:

$$
\eta_{\mathrm{ad}}=\frac{1-\frac{T_{0}^{\mathrm{RO}}}{T_{0}^{\mathrm{SI}}}}{1-\left(\frac{p_{0}^{\mathrm{RO}}}{p_{0}^{\mathrm{SI}}}\right)^{\frac{\gamma-1}{\gamma}}},
$$

where $T_{0}^{\mathrm{RO}}$ and $p_{0}^{\mathrm{RO}}$ are the total temperature and pressure at the rotor blade outlet, respectively, $T_{0}^{\mathrm{SI}}$ and $p_{0}^{\text {SI }}$ are the total temperature and pressure at the stator blade inlet, respectively, and $\gamma$ is the heat capacity ratio. The total temperature and pressure are obtained by

$$
T_{0}=T\left(1+\frac{\gamma-1}{2} M^{2}\right)
$$

and

$$
p_{0}=p\left(1+\frac{\gamma-1}{2} M^{2}\right)^{\frac{\gamma}{\gamma-1}}
$$

respectively, where $T$ is the static temperature, $p$ is the static pressure, and $M$ is the local Mach number, which we extract directly from the CFD solutions. The subscript 0 denotes total (or stagnation) quantities.

The calculation of these performance metrics is completed with time-averaged data. Inflow conditions specified in Figure 2 are applied everywhere in the flow field as the initial condition. The simulation operates for
Table 1 Mesh convergence study: statistics of meshes and results.

\begin{tabular}{|c|c|c|c|}
\hline & $\begin{array}{c}\text { Blade element } \\
\text { size }(\mathrm{mm})\end{array}$ & $\begin{array}{c}\text { Power } \\
(\mathrm{kW})\end{array}$ & $\begin{array}{c}\text { Relative error } \\
(\%)\end{array}$ \\
\hline Mesh 1 & 2.4 & 673.6 & 5.23 \\
\hline Mesh 2 & 1.2 & 701.1 & 1.32 \\
\hline Mesh 3 & 0.6 & 707.8 & 0.39 \\
\hline Mesh 4 & 0.3 & 710.6 & - \\
\hline
\end{tabular}

2.2 revolutions to fully develop the flow field and then the following 3.3 revolutions are used to obtain a timeaveraged solution. The data is extracted with a spatially averaging approach that determines the mean pressure, temperature and Mach number entering and exiting the blade passage. These values are obtained by averaging annular slices on a $0.5 \mathrm{~mm}$ interval that are between $3 \mathrm{~mm}$ and $7 \mathrm{~mm}$ upstream from the leading edge of the stator and downstream from the trailing edge of the rotor. This approach reduces the uncertainty caused by extracting data from a single plane.

For the 3D simulation results presented in the following section, the time-averaged rotor blade pressure contours, vortical structures ( $Q$-criterion [47] isosurfaces), and streamlines in the rotor blade passage are analyzed to locate and rate the magnitude of flow separation, and to identify and analyze recirculation zones and pressure disparity regions.

\section{RESULTS AND DISCUSSION}

\subsection{Mesh convergence study}

We first carry out a mesh convergence study to determine the mesh resolution that yields accurate simulation results of the gas turbine flows in this work. Following the same mesh design strategy in Xu et al. [15, Section 4.1], we construct four meshes of different refinement levels, with blade surface element sizes being $2.4 \mathrm{~mm}, 1.2 \mathrm{~mm}, 0.6 \mathrm{~mm}$ and $0.3 \mathrm{~mm}$, listed in Table 1 . For reference, the rotor blade has a chord length of 20.8 mm. Mesh 3, which has a blade element size of $0.6 \mathrm{~mm}$, employs the same mesh resolution as the one used in $\mathrm{Xu}$ et al. [15, Figure 19].

Simulations are carried out on all four meshes using the baseline blade design with the stage operating at $100 \%$ speed. Table 1 shows the power output for all four cases, with Mesh 3 achieving a relative error of $0.39 \%$ with reference to the most refined case. Given the complexity of the simulation problem, we conclude that a converged solution is achieved at this mesh resolution for the purpose of the present study. For all other simulation cases with rotor blade articulations, the same resolution as Mesh 3 is applied.

\subsection{Effect of off-design rotor speeds}

To understand the effect of off-design rotor speeds, we 


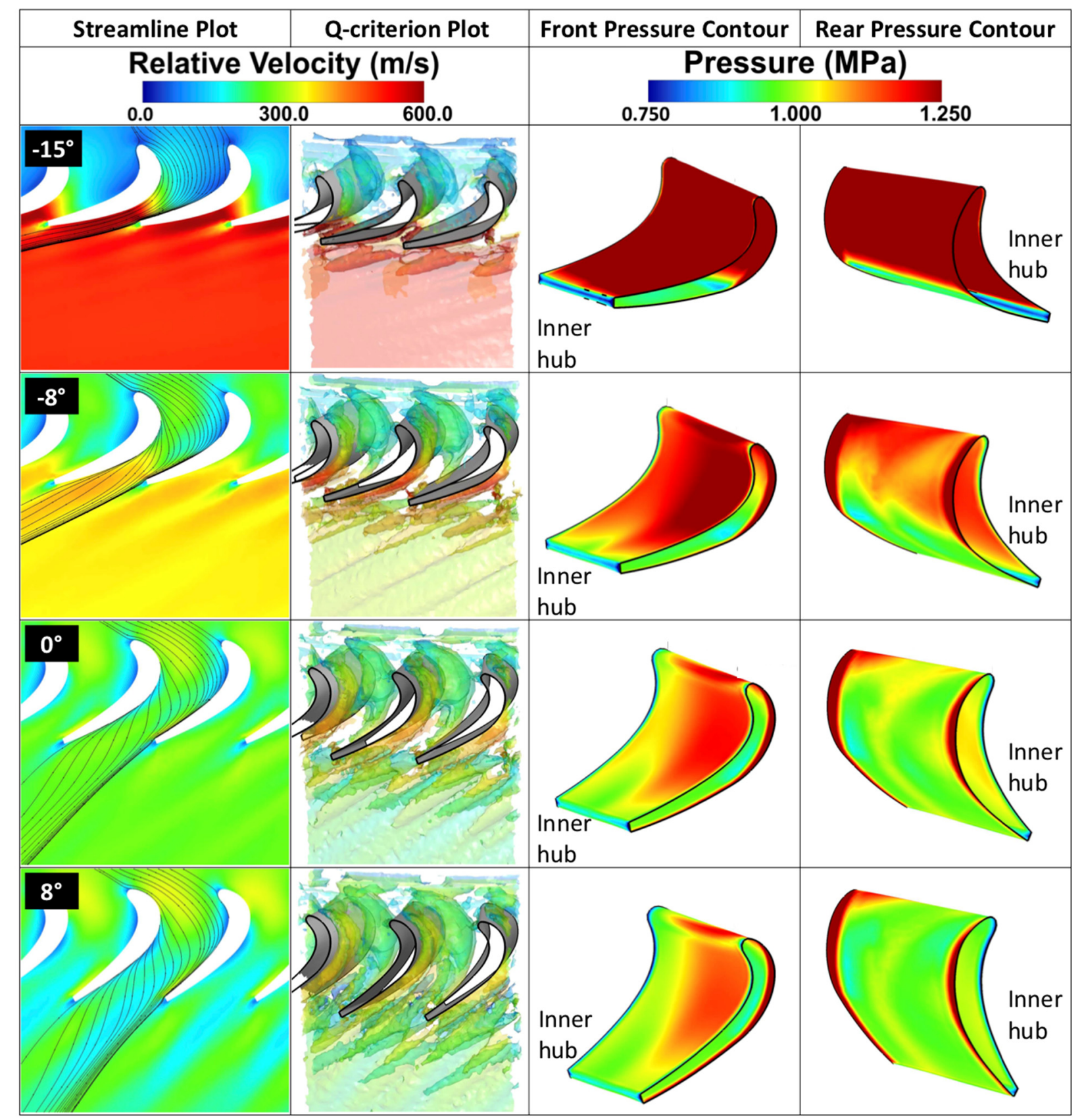

Fig. 4 Streamlines, relative velocity contours, $Q$-criterion isosurfaces, and pressure contours of selected cases operating at $100 \%$ in the moderate articulation range.

examine and compare the flows inside the stage under $100 \%$ and $50 \%$ rotor speeds, simulated with the baseline blade design $\left(0^{\circ}\right)$, shown in Figures 4 and 5, respectively. We focus on the flow separation, which can lead to performance degradation and critical loss of operability. The flow separation is identified in the streamline plots by examining the path of the lines [11]. A line that moves away from the suction side of the blade is said to be detached and separated. The location where the line strays from the suction side is known as the point of separation and the magnitude of flow separation can be derived by how far away the lines move from both the suction and pressure sides. Another indication of increased flow separation is the formation and growth of recirculation zones and slow traveling flow which is illustrated by the curl of the streamlines. Flow separation can also be seen in the $Q$-criterion plots that illustrate the vortical structures of the flow [47, 48]. A vortical structure that is larger in size and colored at a lower speed is the result of increased flow separation. These structures are best observed in the passage near the blade and when the flow exits the passage. Finally, we look at the pressure contour on the blade surfaces to identify adverse pressure gradient that causes the flow separation.

Figures 4 and 5 show that the $0^{\circ}$ case at $50 \%$ speed features higher velocity gradients near the leading edge of the suction side, the trailing edge on the pressure side, and 


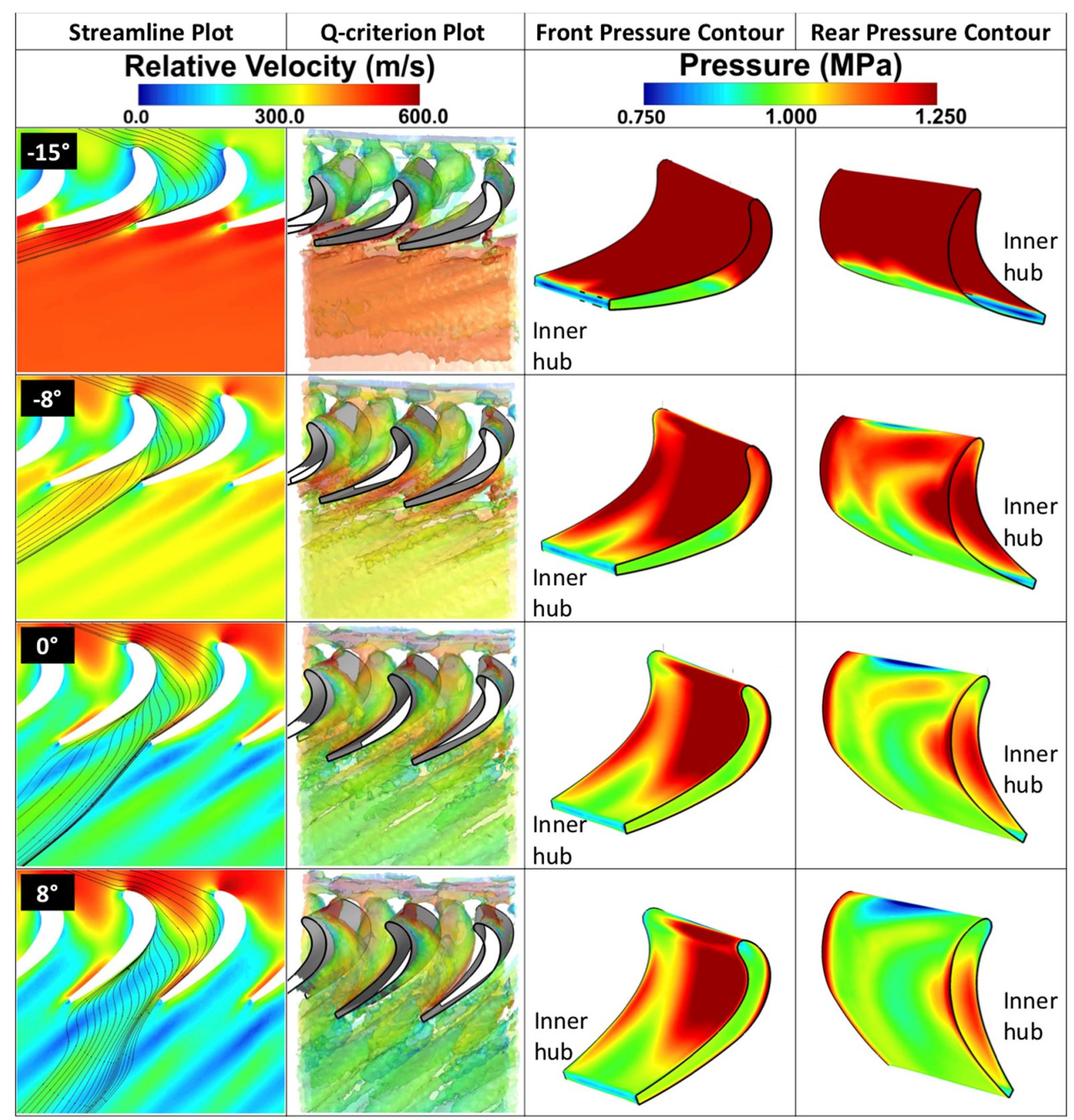

Fig. 5 Streamlines, relative velocity contours, $Q$-criterion isosurfaces, and pressure contours of selected cases operating at $50 \%$ in the moderate articulation range.

in the downstream flow. These gradients amplify high and low velocity regions that imply an increase in flow separation and are shown in the streamline and relative velocity contour plots. Furthermore, this separation leads to increased vortex core regions in the areas of increased velocity gradients as shown in the $Q$-criterion plots. The pressure contours depict a gradient in the opposite direction of the main stream flow at the 50\% operating speed on the suction surface. This addresses the root cause of increased flow separation with operation at an off-design rotor speed. As will become evident in the following sections, this flow separation can adversely influence the gas turbine performance metrics.

\subsection{Moderate rotor articulation}

It was stated in Murugan et al. [6] that the proposed mechanism for blade articulation could have the capability to pitch the blades $\pm 15^{\circ}$ to address the shortcomings discussed in the previous section. Motivated by this, we first primarily focus on exploring the influence of this mechanism within the moderate range of rotor blades.

The influence of rotor blade articulation in the positive angle is discussed by comparing two blade angles at the same rotor speed. Referencing the cases with the rotor at $0^{\circ}$ and $8^{\circ}$, shown in Figures 4 and 5, we observe the increase of flow separation with a positive articulation. We see this increase at both speeds with the point of separation occurring closer to the leading edge and the reduction of flow speed in the passage at $8^{\circ}$. These changes in the flow are reflected in the $Q$-criterion plots 


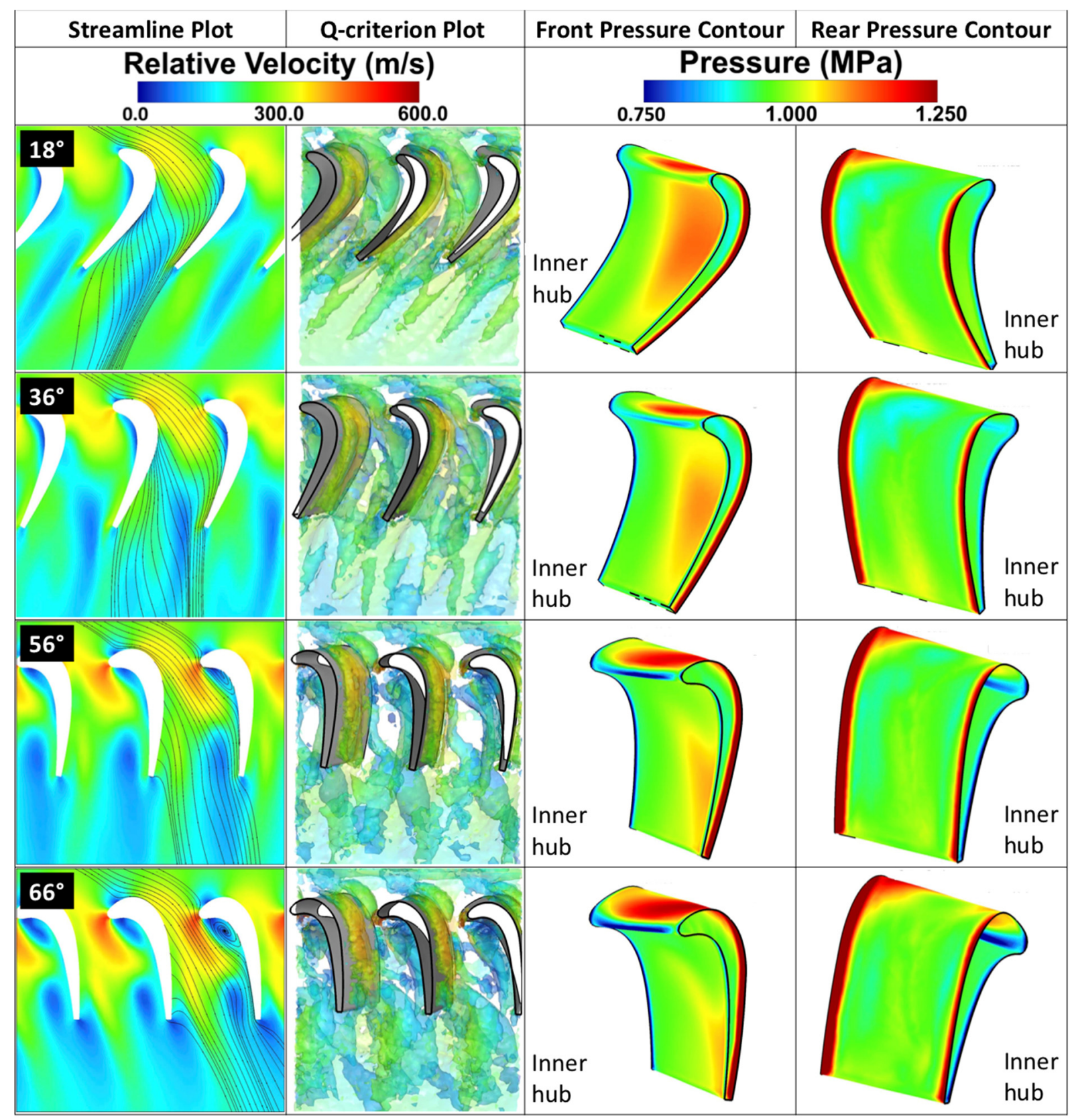

Fig. 6 Streamlines, relative velocity contours, $Q$-criterion isosurfaces, and pressure contours of selected cases operating at $100 \%$ in the extreme articulation range.

by the growth of vortical structures in the passage of the blade and downstream. The pressure contours support this increase of flow separation by the increased adverse pressure gradients shown near the leading edge on the suction side of the blade. All of these trends stay true for other positive articulations in the moderate range.

A negative articulation from $0^{\circ}$ to $-8^{\circ}$ reduces the flow separation and appears to be inverse of the $8^{\circ}$ case. This reduction is seen by the delay of the location of the separation point with the increase of passage flow velocity at both the rotor speeds. The $Q$-criterion plots depict this reduction by the shrinking of isosurfaces in the middle of the passage and at the trailing edge. The pressure contour plots support this reduced flow separation by illustrating the favorable pressure gradient all the way along the blade surface. We note that with a negative articulation, the flow exits the rotor passage at a larger velocity than when it enters, which is clearly contradictory to the baseline design or the positive articulation at $8^{\circ}$. At $-15^{\circ}$, flow separation continues to be minimized as depicted in the streamline plots with increased suction side attachment in both speeds and in the pressure contours with a more homogeneous distribution. This minimization is illustrated in the $Q$-criterion plots with the reduction of isosurfaces. A further negative articulation induces a larger favorable pressure gradient observed on the suction side, causing the flow to be more greatly accelerated in the rotor passage. At both rotor speeds, the flow velocities exiting the rotor passages reach peak values, suggesting that a large portion of the flow energy 


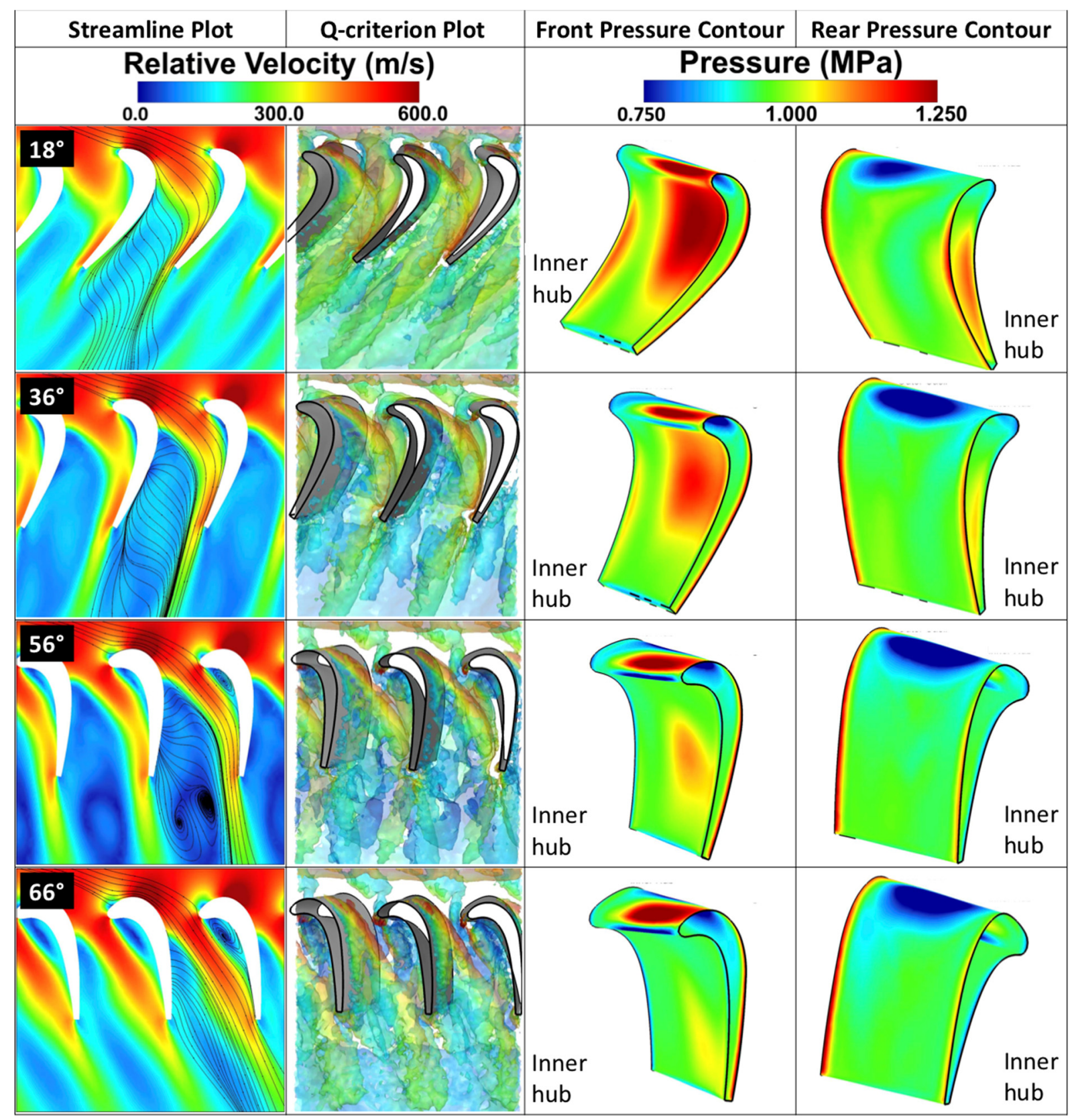

Fig. 7 Streamlines, relative velocity contours, Q-criterion isosurfaces, and pressure contours of selected cases operating at $50 \%$ in the extreme articulation range.

is lost in the form of fluid kinetic energy, rather than being converted into shaft power.

\subsection{Extreme rotor articulation}

To further the understanding of the impact of blade articulation on turbine performance, we explore an extreme range of rotor pitch angles in this section. Previously in the literature, Suder et al. [49] and Ainley and Mathieson [50] investigated an articulation range of up to $80^{\circ}$ to explore the effect of blade articulation on the stage losses. Extreme rotor articulations contradict typical turbomachinery conditions but provide valuable insight into the effects of a wide range of articulation angles. These effects include major flow dynamic variations within the rotor passage such as extensive separations and increased vortices. The discussion in what follows elaborates on such flow variations illustrated in Figures 6 and 7.

The first extreme case is the rotor position of $18^{\circ}$. Comparing to the $8^{\circ}$ case, this further positive articulation leads to increased flow separation. We observe this increase with the more aggressive flow detachment at both operating speeds because the streamlines increase their curl to travel downwards from the suction side rather than moving towards the pressure side to reattach to the rest of the flow. This behavior provides insight that the flow nearly passes through the passage without facing a significant turn in direction, which can also be observed in the pressure contours with the decrease of the pressure 


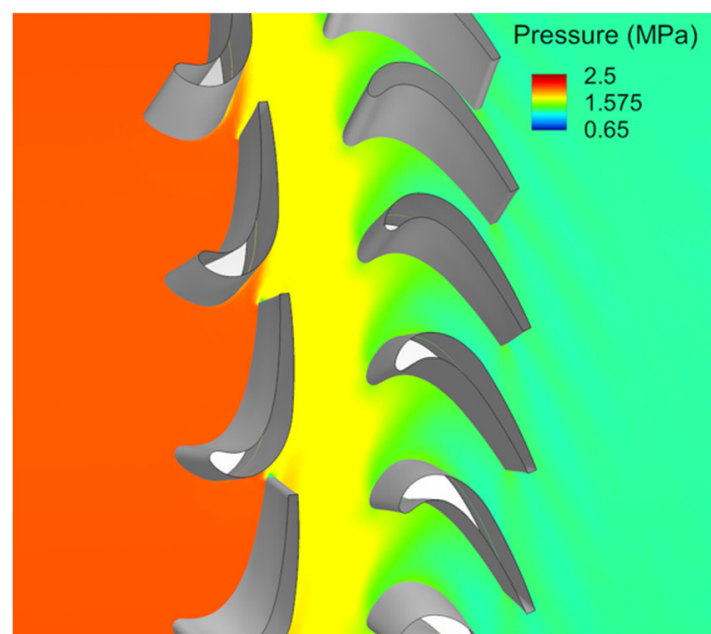

(a) Total pressure

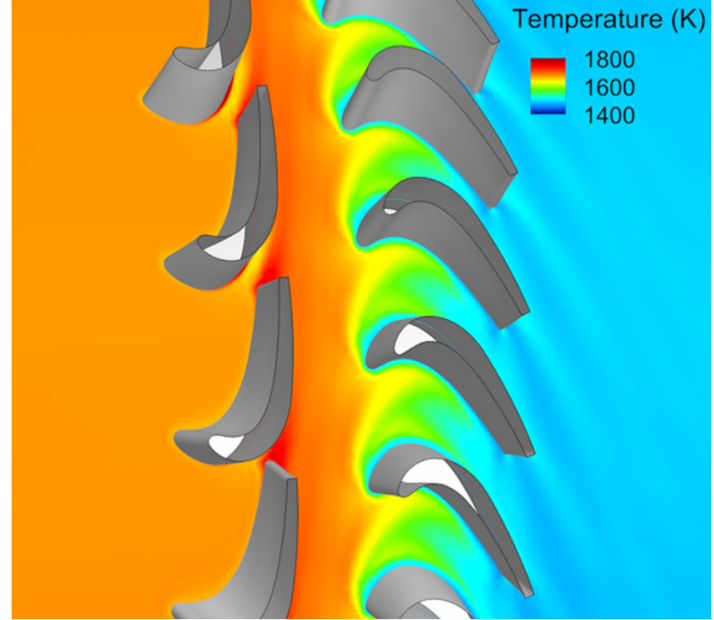

(b) Total temperature

Fig. 8 Contours of the time-averaged total pressure and total temperature around the turbine blades.

gradient on the pressure side of the blade. At both speeds, the vortex size increases for the flow exiting the rotor blade supporting the increase of flow separation.

However, at $36^{\circ}$, these behaviors are slightly modified because of how the flow impacts the rotor blade. The stagnation point is no longer at the leading edge of the blade but shifted towards the suction surface as seen in the streamline and pressure plots. This change in contact is depicted in the variation of the locations of highest pressures in the pressure contours. The increased flow separation can be seen through the streamlines deviating further from the geometry and increase of reduced speed zones at both rotor speeds. The $50 \%$ speed also begins to exhibit a new flow behavior that is not seen in the previous blade positions. The streamlines curl towards the opposite direction of main-stream flow, suggesting the presence of reverse flows near the suction side and extensive flow separations. As a result, a shear layer can be clearly observed in the middle of the passage. This increased flow separation is illustrated by the enlargement of vortical structures in both the passage and downstream flow, shown in the $Q$-criterion plots.

The trends seen in the $36^{\circ}$ case do not apply to the $56^{\circ}$ case as the rotor's articulation begins to direct the flow in a new manner. The extreme positive articulation creates a larger angle of attach between flow and the pressure surface, causing a notable recirculation zone on the pressure side of the blade. Compared with previous cases, the flow now exits with a rightwards direction, and the recirculation downstream of the suction side has reached a peak in $50 \%$ speed plot. Large recirculation zones begin to form downstream of the blade passage, which can also be seen from the enlargement of $Q$ criterion isosurfaces. The huge adverse pressure gradients on both the pressure and suction sides explain the extensive flow separation in the case of $50 \%$ rotor speed. The trends of the $56^{\circ}$ largely apply to the $66^{\circ}$ case with a small variation. While the flow separation on the pressure side persists and intensifies, suction side appears to have more attached flow as the downstream recirculation zones are not present and the streamlines are more aligned.

\subsection{Impact on turbine performance metrics}

Following the discussion in Section 2.3, we extract the time-averaged total temperature and total pressure at the stator inlet and rotor outlet, and calculate the efficiency for all the cases we presented in the previous sections. The contours of time-averaged total pressure and total temperature of the baseline design at $100 \%$ rotor speed are show in Figure 8. A summary of the performance metrics versus rotor blade articulation angle is presented in Figure 9. Figure 9(a) illustrates the relationship of degrading efficiency with positive angle articulation. This trend is consistent with the trend of flow field changes in our previous flow analysis, as when the articulation angles change from negative to positive, flow separations generally increase and therefore cause larger losses. Figure 9(b) suggests that the shaft power output increases when articulation angles change from negative to positive. From the fluid velocity contour, it is obvious that with negative articulation angles, the flow greatly accelerates in the rotor passage and exits with larger kinetic energy; therefore less energy is transformed into shaft power. As the blade articulation angle goes positive, flow exits at a smaller velocity and thus more flow energy is converted to provide shaft power. It is particularly interesting to note the plateau region in turbine power past $+10^{\circ}$ articulation. This suggests that in the blade angle range of $+10^{\circ}$ to $+55^{\circ}$, only engine efficiency is severely influenced, while the power output is maintained roughly constant. This behavior can be used to guide the control of rotor articulation for a good combination of power and efficiency.

\section{CONCLUSIONS AND FUTURE WORK}

This study focuses on using advanced, moving-domain 


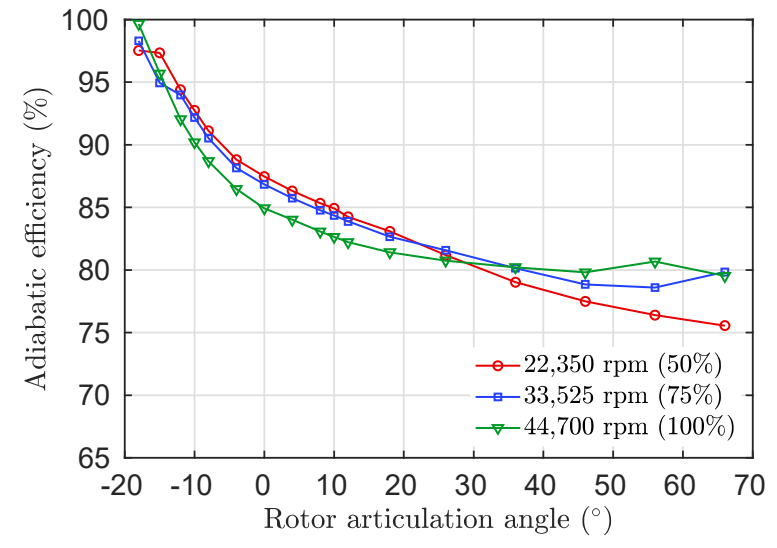

(a) Adiabatic efficiency

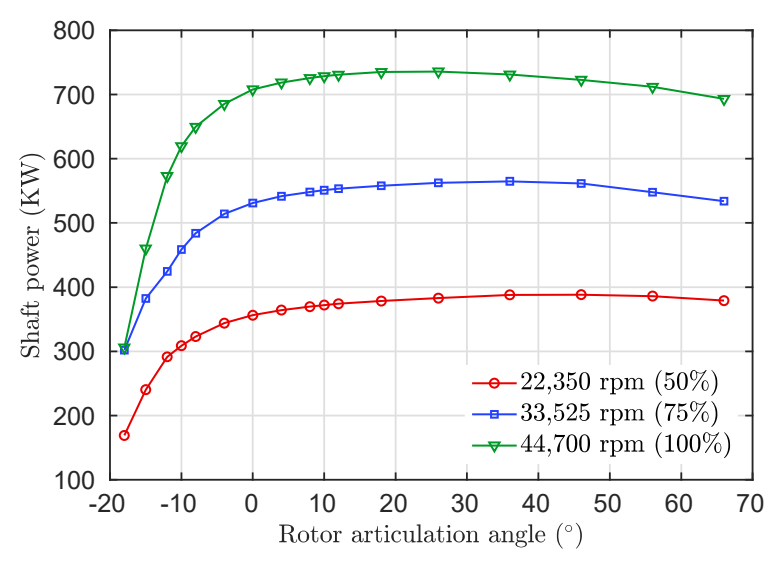

(b) Shaft power

Fig. 9 Gas turbine engine performance metrics dependencies on rotor blade articulation angle during operation.

compressible flow modeling and simulation to better understand the performance of a gas turbine stage for a variety of operating conditions. These operating conditions include large variations in the blade angle on the rotor sections, as well as variations in the rotor speed. Given that airfoil-like surfaces are employed in the gasturbine model, changing the relative air speed and angle of attack leads to the flow transitioning from fully attached, to partially attached, to fully separated regime. As a result, the present application requires a methodology that automatically adjusts to the flow regime present in the problem, and thus makes classical approaches problematic. On the other hand, a combination of a stabilized formulation, which may be motivated through variational multiscale modeling of turbulence, and weakly enforced no-slip boundary conditions, which act as a near-wall model without explicit dependence on the boundary-layer parameterization, provides this flexibility and robustness with respect to the flow regime. This, in turn, leads to a successful deployment of our compressible-flow simulation framework for the present application that requires system exploration for large variations of input parameters.

The simulation results in this work illustrate the influences of rotor articulation angles on flow separations and changes in gas turbine stage thermal efficiency and power output. It is evident that the positive articulation results in increased flow separation and pressure losses leading to a significant degradation in gas turbine efficiency, while the negative articulation performs in the opposite way. In summary, this study provides much needed information on the aerodynamic characteristics in the turbine stage featuring the incidence-tolerant blade. It determines the possible limits on articulation and expected performance benefits that can serve to improve the design of a VSPT, which could change the blade angles according to real-time operating conditions. In the future, we plan to continue to further explore and understand the relationship between blade articulation and gas turbine engine performance by investigating synchronous stator/rotor articulation.

\section{ACKNOWLEDGMENTS}

N. Kozak was supported by the 2018 HPC Internship Program at ARL Vehicle Technology Directorate. F. Xu, Y. Bazilevs and M.-C. Hsu were partially supported by the ARO Grant No. W911NF-14-1-0296. The authors gratefully acknowledge the Department of Defense High Performance Computing Modernization Program Office (HPCMP) for the computing resources on Excalibur, Onyx, and Centennial. All this support is gratefully acknowledged.

\section{REFERENCES}

1. Welch, G. E., "Assessment of aerodynamic challenges of a variable-speed power turbine for large civil tilt-rotor application," In Proceedings of the 66th American Helicopter Society International Annual Forum (AHS Forum 66) (2010).

2. Murugan, M., Booth, D., Ghoshal, A., Thurman, D. and Kerner K., "Concept study for adaptive gas turbine rotor blade," The International Journal of Engineering and Science, 4, pp. 10-17 (2015).

3. Murugan, M., Ghoshal, A., Xu, F., Hsu, M.-C., Bazilevs, Y., Bravo, L. and Kerner, K., "Analytical study of articulating turbine rotor blade concept for improved off-design performance of gas turbine engines," Journal of Engineering for Gas Turbines and Power, 139, pp. 102601 (2017).

4. Walker, G. J., "The role of laminar-turbulent transition in gas turbine engines: A discussion," Journal of Turbomachinery, 115, pp. 207-216 (1991).

5. Howard, S. A., "Rotor dynamic feasibility of a conceptual variable-speed power turbine propulsion system for large civil tilt-rotor applications," In Proceedings of the 68th American Helicopter Society International Annual Forum (AHS Forum 68) (2012).

6. Murugan, M., Ghoshal, A. and Bravo, L., "Adaptable articulating axial-flow compressor/turbine rotor 
blade," US Patent Application 20180066671 A1 (2017).

7. Le, B. and Alvin, T., "Variable stator vanes," US Patent 3237918A (1963).

8. Weiler, W., "Variable stator cascades for axial-flow turbines of gas turbine engines," US Patent 4314791A (1978).

9. Olive, C. E., "Control of variable stator vanes," US Patent 5024580A (1989).

10. Brethouwer, G., "The effect of rotation on rapidly sheared homogeneous turbulence and passive scalar transport. Linear theory and direct numerical simulation," Journal of Fluid Mechanics, 542, pp. 305-342 (2005).

11. Acharya, S. and Mahmood, G., "Turbine blade aerodynamics," In The Gas Turbine Handbook, chapter 4.3, National Energy Technology Laboratory, (2006).

12. Corke, T. C. and Thomas, F. O., "Dynamic stall in pitching airfoils: aerodynamic damping and compressibility effects," Annual Review of Fluid Mechanics, 47, pp. 479-505 (2015).

13. Wirkowski, P., "Influence of the incorrect settings of axial compressor inlet variable stator vanes on gas turbine engine work parameters," Journal of KONES, Powertrain and Transport, 19, pp. 483-489 (2015).

14. Wang, Z., Li, J., Fan, K. and Li. S., "The off-design performance simulation of marine gas turbine based on optimum scheduling of variable stator vanes," Mathematical Problems in Engineering, 2017, pp. 2671251 (2017).

15. Xu, F., Moutsanidis, G., Kamensky, D., Hsu, M.-C., Murugan, M., Ghoshal, A. and Bazilevs, Y., "Compressible flows on moving domains: Stabilized methods, weakly enforced essential boundary conditions, sliding interfaces, and application to gasturbine modeling," Computers \& Fluids, 158, pp. 201-220 (2017).

16. Hughes, T. J. R. and Tezduyar, T. E., "Finite element methods for first-order hyperbolic systems with particular emphasis on the compressible Euler equations," Computer Methods in Applied Mechanics and Engineering, 45, pp. 217-284 (1984).

17. Hughes, T. J. R., Franca, L. P. and Mallet, M., “A new finite element formulation for computational fluid dynamics: I. Symmetric forms of the compressible Euler and Navier-Stokes equations and the second law of thermodynamics," Computer Methods in Applied Mechanics and Engineering, 54, pp. 223234 (1986).

18. Hughes, T. J. R. and Mallet, M., “A new finite element formulation for computational fluid dynamics: III. The generalized streamline operator for multidimensional advective-diffusive systems," Computer Methods in Applied Mechanics and Engineering, 58, pp. 305328 (1986).
19. Hughes, T. J. R., Franca, L. P. and Mallet, M., "A new finite element formulation for computational fluid dynamics: VI. Convergence analysis of the generalized SUPG formulation for linear timedependent multi-dimensional advective-diffusive systems," Computer Methods in Applied Mechanics and Engineering, 63, pp. 97-112 (1987).

20. Hauke, G. and Hughes, T. J. R., "A unified approach to compressible and incompressible flows," Computer Methods in Applied Mechanics and Engineering, 113, pp. 389-396 (1994).

21. Hauke, G. and Hughes, T. J. R., "A comparative study of different sets of variables for solving compressible and incompressible flows," Computer Methods in Applied Mechanics and Engineering, 153, pp. 1-44 (1998).

22. Hauke, G., "sabilizing matrices for the computation of compressible flows in primitive variables," Computer Methods in Applied Mechanics and Engineering, 190, pp. 6881-6893 (2001).

23. Tezduyar, T. E. and Park, Y. J., "Discontinuity capturing finite element formulations for nonlinear convection-diffusion-reaction equations," Computer Methods in Applied Mechanics and Engineering, 59, pp. 307-325 (1986).

24. Hughes, T. J. R., Mallet, M. and Mizukami, A., “A new finite element formulation for computational fluid dynamics: II. Beyond Simple SUPG," Computer Methods in Applied Mechanics and Engineering, 54, pp. 341-355 (1986).

25. Hughes, T. J. R. and Mallet, M., "A new finite element formulation for computational fluid dynamics: IV. A discontinuity-capturing operator for multidimensional advective-diffusive systems," Computer Methods in Applied Mechanics and Engineering, 58, pp. 329-339 (1986).

26. Tezduyar, T. E., "Finite element methods for fluid dynamics with moving boundaries and interfaces," In E. Stein, R. De Borst, and T. J. R. Hughes, editors, Encyclopedia of Computational Mechanics, Volume 3: Fluids, chapter 17, John Wiley \& Sons (2004).

27. Rispoli, F., Saavedra, R., Corsini, A. and Tezduyar, T. E., "Computation of inviscid compressible flows with the V-SGS stabilization and YZ $\beta$ shockcapturing," International Journal for Numerical Methods in Fluids, 54, pp. 695-706 (2007).

28. Rispoli, F., Saavedra, R., Menichini, F. and Tezduyar, T. E., "Computation of inviscid supersonic flows around cylinders and spheres with the V-SGS stabilization and YZ $\beta$ shock-capturing," Journal of Applied Mechanics, 76, pp. 021209 (2009).

29. Rispoli, F., Delibra, G., Venturini, P., Corsini, A., Saavedra, R. and Tezduyar, T. E., "Particle tracking and particle-shock interaction in compressible-flow computations with the V-SGS stabilization and YZ $\beta$ shock-capturing," Computational Mechanics, 55, pp. 1201-1209 (2015). 
30. Takizawa, K., Tezduyar, T. E. and Otoguro, Y., "Stabilization and discontinuity-capturing parameters for space-time flow computations with finite element and isogeometric discretizations," Computational Mechanics, 62, pp. 1169-1186 (2018).

31. Bazilevs, Y. and Hughes, T. J. R., "Weak imposition of Dirichlet boundary conditions in fluid mechanics," Computers \& Fluids, 36, pp.12-26 (2007).

32. Bazilevs, Y., Michler, C., Calo, V. M. and Hughes, T. J. R., "Weak Dirichlet boundary conditions for wallbounded turbulent flows," Computer Methods in Applied Mechanics and Engineering, 196, pp. 48534862 (2007).

33. Bazilevs, Y., Michler, C., Calo, V. M. and Hughes, T. J. R., "Isogeometric variational multiscale modeling of wall-bounded turbulent flows with weakly enforced boundary conditions on unstretched meshes," Computer Methods in Applied Mechanics and Engineering, 199, pp. 780-790 (2010).

34. Bazilevs, Y. and Akkerman, I., "Large eddy simulation of turbulent Taylor-Couette flow using isogeometric analysis and the residual-based variational multiscale method," Journal of Computational Physics, 229, pp. 3402-3414 (2010).

35. Hsu, M.-C., Akkerman, I. and Bazilevs, Y., "Wind turbine aerodynamics using ALE-VMS: Validation and the role of weakly enforced boundary conditions," Computational Mechanics, 50, pp. 499-511 (2012).

36. Bazilevs, Y. and Hughes, T. J. R., "NURBS-based isogeometric analysis for the computation of flows about rotating components," Computational Mechanics, 43, pp. 143-150 (2008).

37. Hsu, M.-C., Akkerman, I. and Bazilevs, Y., "Finite element simulation of wind turbine aerodynamics: Validation study using NREL Phase VI experiment," Wind Energy, 17, pp. 461-481 (2014).

38. Hsu, M.-C. and Bazilevs, Y., "Fluid-structure interaction modeling of wind turbines: simulating the full machine," Computational Mechanics, 50, pp. 821-833 (2012).

39. Xu, F., Bazilevs, Y. and Hsu, M.-C., "Immersogeometric analysis of compressible flows with application to aerodynamic simulation of rotorcraft," Mathematical Models and Methods in Applied Sciences, 29, pp. 905-938 (2019).

40. Chung, J. and Hulbert, G. M., "A time integration algorithm for structural dynamics with improved numerical dissipation: The generalized- $\alpha$ method," Journal of Applied Mechanics, 60, pp. 371-75 (1993).

41. Jansen, K. E., Whiting, C. H. and Hulbert, G. M., "A generalized- $\alpha$ method for integrating the filtered Navier-Stokes equations with a stabilized finite element method," Computer Methods in Applied Mechanics and Engineering, 190, pp. 305-319 (2000).

42. Bazilevs, Y., Calo, V. M., Hughes, T. J. R. and Zhang, Y., "Isogeometric fluid-structure interaction: theory, algorithms, and computations," Computational Mechanics, 43, pp. 3-37 (2008).

43. Shakib, F., Hughes, T. J. R. and Johan, Z., "A multielement group preconditioned GMRES algorithm for nonsymmetric systems arising in finite element analysis," Computer Methods in Applied Mechanics and Engineering, 75, pp. 415-456 (1989).

44. Hsu, M.-C., Wang, C., Herrema, A. J., Schillinger, D., Ghoshal, A. and Bazilevs, Y., "An interactive geometry modeling and parametric design platform for isogeometric analysis," Computers and Mathematics with Applications, 70, pp. 1481-1500 (2015).

45. Schobeiri, M., Turbomachinery Flow Physics and Dynamic Performance, Springer-Verlag Berlin Heidelberg (2005).

46. Logan, E. Jr., and Roy, R., Handbook of Turbomachinery. CRC Press, Boca Raton, FL, 2nd edition (2003).

47. Jeong, J. and Hussain, F., "On the identification of a vortex," Journal of Fluid Mechanics, 285, pp. 69-94 (1995).

48. Hunt, J. C. R., Wray, A. A. and Moin, P., "Eddies, streams, and convergence zones in turbulent flows," In Proceeding of the Summer Program 1988, pp. 193-208. Center for Turbulence Research (1988).

49. Suder, K., Durbin, K., Giel, P., Poinsatte, P., Thrurman, P. and Ameri, A., "Variable speed turbine technology development and demonstration," In Proceedings of the 74th American Helicopter Society International Annual Forum (AHS Forum 74) (2018).

50. Ainley, D. G. and Mathieson, G. C. R., "A method of performance estimation for axial-flow turbines," Technical report, Aeronautical Research Council Reports and Memoranda, no. 2974 (1951).

(Manuscript received August 20, 2019, accepted for publication September 24, 2019.) 